\title{
SATURATION OF THE APPROXIMATION BY EIGENFUNCTION EXPANSIONS ASSOCIATED WITH THE LAPLACE OPERATOR
}

\author{
SATORU IGARI *)
}

(Received January 22, 1970)

1. Let $n \geqq 2$ and $\Omega$ be an open domain in the $n$-dimensional Euclidean space $\boldsymbol{R}^{n}$. Suppose that $u_{k}(x), k=1,2,3, \cdots$ are eigenfunctions of the Laplace operator $\Delta$ and $\lambda_{k}$ are the corresponding eigenvalues, that is,

$$
\Delta u_{k}(x)+\lambda_{k} u_{k}(x)=0 \text { in } \Omega .
$$

We assume that $\left\{u_{k}(x)\right\}_{k=1}^{\infty}$ is a complete orthonormal system in $L^{2}(\Omega)$, and furthermore $\lambda_{k}$ are non-decreasing and tend to infinity. These assumptions will be satisfied if we impose some boundary conditions on eigenfunctions and $\Omega$.

For a function $f$ in $L^{2}(\Omega)$ let

$$
f \sim \sum_{k=1}^{\infty} f_{k} u_{k}(x)
$$

he the Fourier expansion, where

$$
f_{k}=\int_{\Omega} f(x) \overline{u_{k}(x)} d x
$$

We denote the $\lambda$-th $R\left(\lambda_{i}, \delta\right)$ mean by

$$
s_{\lambda}^{i}(f, x)=\sum_{\lambda_{k}<\lambda}\left(1-\begin{array}{c}
\lambda_{k} \\
\lambda
\end{array}\right)^{\delta} f_{k} u_{k}(x) .
$$

$f(x)$ is said to be regulated at $x$ if there exists an approximate identity $\left\{\boldsymbol{\phi}_{i}(x)\right\}$ of infinitely differentiable functions with supports contained in $\{x ;|x| \leqq \varepsilon\}$ such that $f * \varphi_{c}(x)$ tends to $f(x)$.

Let $\alpha=(n-1) / 2$ be the critical index and denote by \|\|$_{k}$ the supremum

\footnotetext{
*) Supported in part by the Sakkokai Foundation.
} 
norm on the set $K$. Our aim is to prove the following.

THEOREM. Let $D$ be an open subdomain of $\Omega, f$ be a function in $L^{2}(\Omega)$ regulated in $D$ and $\delta \geqq \alpha+2$.

(i) It holds that

$$
\left\|s_{\lambda}^{s}(f)-f\right\|_{K}=o(1 / \lambda)
$$

as $\lambda \rightarrow \infty$ for every compact set $K$ in $D$, if and only if $f$ is harmonic in $D$.

(ii) It holds that

$$
\left\|s_{\lambda}^{\hat{o}}(f)-f\right\|_{K}=O(1 / \lambda)
$$

as $\lambda \rightarrow \infty$ for every compact set $K$ in $D$, if and only if $\Delta f$ in the sense of distribution is bounded in every compact set of $D$.

REMARK. Let $\delta \geqq \alpha$ and assume the condition of (i). Then we have

$$
\left\|s_{\lambda}^{\hat{o}}(f)-f\right\|_{K}=o\left(\sqrt{ }^{\alpha-\hat{o}}\right)
$$

as $\lambda \rightarrow \infty$ for every compact set $K$ in $D$. This inequality is valid under the hypothesis of (ii) if $2>\delta-\alpha \geqq 0$

2. The local saturation problem for trigonometric expansions of a variable is studied by [3], for example, but for our case a difficulty arises mainly from the fact that we fail to find any (quasi-) positive summability kernels like the Cesàro or the Poisson kernels, and some different devices will be needed.

Let $\delta>-1$ and $x$ be any point in $\Omega$. If $R>0$ is so small that the sphere $S(x, R)$ of radius $R$ with the center at $x$ is contained in $\Omega$, then we have

$$
s_{\lambda}^{\delta}(f, x)=v_{i}^{\delta, R}(f, x)+w_{\lambda}^{\delta_{\lambda}, \boldsymbol{R}}(f, x),
$$

where

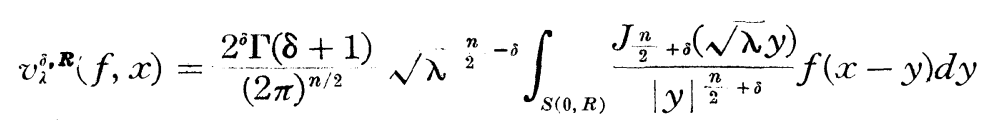

and

$$
w_{k}^{\delta, R}(f ; x)=2^{8} \Gamma(\delta+1) \sqrt{\lambda^{\frac{n}{3}-8}} \sum_{k=1}^{\infty} f_{k} u_{k}(x) \frac{1}{\sqrt{\lambda_{k} \frac{n}{2}-1}} \int_{n}^{\infty} J_{\frac{n}{2}+\delta}(\sqrt{\lambda r}) J_{\frac{n}{2}-1}\left(\sqrt{\lambda_{k}} r\right) r^{-s} d r
$$


(see $[4 ;$ p. 205]).

The order of $w_{\lambda}^{\delta, R}(f, x)$ is given in [2] and [4], but we shall need more accurate estimation.

LEMMA 1. If $f \in L^{2}(\Omega), \delta>0$ and $K$ is a compact set in $\Omega$, then

$$
\left\|w_{\lambda}^{\hat{o}, R}(f)\right\|_{K}=o\left({\sqrt{\lambda^{-\alpha-o}}}^{-}\right.
$$

as $\lambda \rightarrow \infty$ for every $R$ such that $0<R<\operatorname{dis}\left(K, \Omega^{c}\right)$.

Proof. Put

$$
I_{k}^{\lambda}=\int_{R}^{\infty} J_{\frac{n}{2}+\delta}(\sqrt{ } \lambda r) J_{\frac{n}{2}-1}\left(\sqrt{ } \lambda_{k} r\right) r^{-\hat{o}} d r
$$

By integration by parts and an asymptotic formula for the Bessel function we have

$$
\left|I_{k}{ }^{2}\right|<A \lambda^{-\frac{1}{4}} \lambda_{k}^{-1}
$$

for all positive $\lambda$ and $\lambda_{k}$,

$$
\left|I_{k}{ }^{2}\right|<A \frac{\lambda^{-\frac{3}{4}} \lambda_{k}^{\frac{1}{4}}}{\sqrt{\bar{\lambda}}-\sqrt{\lambda_{k}}}+A \lambda^{-\frac{3}{4}} \lambda_{k}^{-\frac{1}{4}} \quad\left(\lambda_{k}<\lambda\right),
$$

and

$$
\left|I_{\kappa}^{\lambda}\right|<A \frac{\lambda^{\frac{1}{4}} \lambda_{k}^{-\frac{3}{4}}}{\sqrt{\lambda_{k}-\sqrt{\lambda}}}+A \lambda^{-\frac{1}{4}} \lambda_{k}^{-\frac{3}{4}} \quad\left(\lambda_{k}>\lambda\right),
$$

where $A$ denotes a constant and may be different in each occasion (see $[4 ; \mathrm{p}$. 202]).

Divide the summation $w_{\lambda}^{\delta, R}(f, x)$ into three sums; $\sqrt{\lambda_{k}}<\sqrt{\lambda}-1, \mid \sqrt{\lambda_{k}}$ $-\sqrt{\lambda} \mid \leqq 1$ and $\sqrt{\lambda}+1<\sqrt{\lambda_{k}}$, and denote by $\Sigma_{1}, \Sigma_{2}, \quad \Sigma_{3}$ the corresponding terms respectively.

In $\Sigma_{2}$ we have $\left|I_{k}^{\lambda}\right|<A \lambda^{-\frac{1}{2}}$. By Schwarz' inequality

$$
\begin{aligned}
& \left|\Sigma_{2}\right| \leqq A \sqrt{\lambda^{-\delta}} \sum_{\left|\mathcal{V}^{2}-\sqrt{\lambda}\right| \leqq 1}\left|f_{k} u_{k}(x)\right| \\
& \leqq A \sqrt{\lambda}\left(\sum\left|f_{k}\right|^{2}\right)^{1 / 2}\left(\sum\left|u_{k}(x)\right|^{2}\right)^{1 / 2} .
\end{aligned}
$$

But 


$$
\sum_{\mid \sqrt{\lambda_{k}-M \mid \leqq 1}}\left|u_{k}(x)\right|^{2}=O\left(M^{n-1}\right)
$$

uniformly on every compact set (see [1]). Thus

$$
\Sigma_{2}=o\left(\sqrt{\lambda^{-\alpha-\bar{o}}}\right) \text {. }
$$

For $\Sigma_{1}$ we have

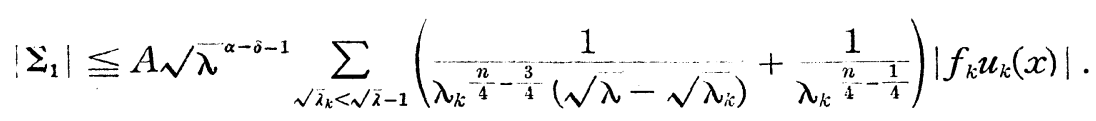

The first term on the right hand side is dominated by

$$
\begin{aligned}
& A \sqrt{\lambda^{\alpha-\delta-1}}+A \sqrt{\bar{\lambda}^{\alpha-\hat{o}-1}}\left(\sum_{k>N}\left|f_{k}\right|^{2}\right)^{1 / 2}\left(\sum_{\sqrt{\lambda_{k}<\sqrt{\lambda}-1}} \frac{\left|u_{k}(x)\right|^{2}}{\lambda_{k}{ }^{\frac{n}{2}-\frac{3}{2}}\left(\sqrt{\lambda}-\sqrt{\lambda_{k}}\right)^{2}}\right)^{1 / 2}
\end{aligned}
$$

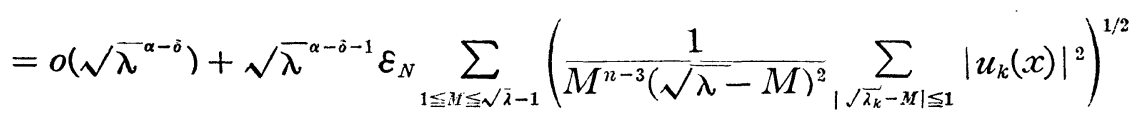

$$
\begin{aligned}
& =o\left({\sqrt{\lambda^{\alpha-\delta}}}^{\alpha}+\sqrt{\bar{\lambda}^{\alpha-\hat{o}} \varepsilon_{N}^{\prime}},\right.
\end{aligned}
$$

where $N$ is an arbitrarily fixed number and $\varepsilon_{N}, \varepsilon_{N} \rightarrow 0$ as $N \rightarrow \infty$. The second term is $o\left(\sqrt{\lambda}^{\alpha-\hat{o}}\right)$ in the similar way. Hence $\Sigma_{1}=o\left(\sqrt{\lambda^{\alpha-j}}\right)$.

$\Sigma_{3}$ is bounded by

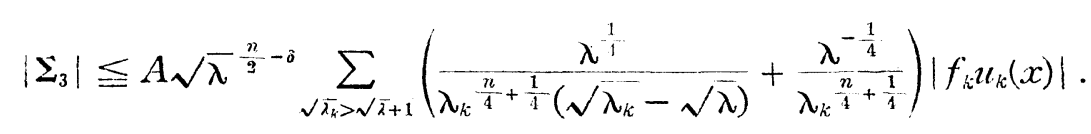

The first term on the right hand side is dominated by

$$
A \sqrt{\lambda^{-\frac{n}{2}-\hat{\sigma}+\frac{1}{2}}}\left(\sum\left|f_{k}\right|^{2}\right)^{1 / 2}\left(\sum \frac{\left|u_{k}(x)\right|^{2}}{\lambda_{k}^{\frac{n+1}{2}}\left(\sqrt{\lambda_{k}}-\sqrt{\lambda}\right)^{2}}\right)^{1 / 2}
$$

which is $o\left(\sqrt{ } \lambda^{\alpha-\hat{o}}\right)$ by the same way as in the $\Sigma_{1}$ case. The order of the second term is rather easily estimated and $o\left(\sqrt{ } \lambda^{-\alpha-\delta}\right)$. Thus the lemma is proved.

3. ProOF of (i). First assume that $f$ is harmonic in $D$. Let $K$ be a compact set contained in $D$. If $x \in K$ and $0<R<\operatorname{dis}\left(K, \Omega^{c}\right)$, then

$$
v_{\lambda}^{\dot{o} . R}(f, x)=c \sqrt{\lambda^{-\frac{n}{2}-\hat{o}}} \int_{\|}^{k} J_{\frac{n}{2}+\hat{j}}(\sqrt{\lambda} r) r^{-\frac{n}{2}-\hat{o}-1}\left(\int_{|\omega|+1} f(x-r \omega) d \omega\right) d r
$$




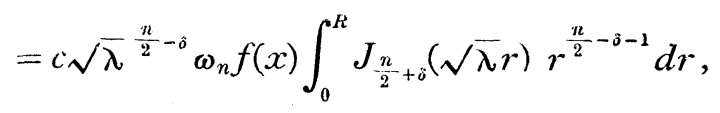

where $c=2^{\delta} \Gamma(\delta+1) / \sqrt{2 \pi}^{n}$ and $\omega_{n}$ is the surface area $\sqrt{2 \pi}^{n} / \Gamma(n / 2)$ of the unit ball in $\boldsymbol{R}^{n}$. If $\delta>\alpha-1$,

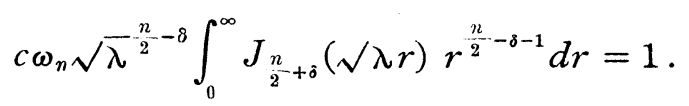

Thus

$$
v_{\lambda}^{\delta, R}(f, x)-f(x)=-c \omega_{n} \sqrt{\lambda^{-\frac{n}{2}-\delta}} f(x) \int_{R}^{\infty} J_{\frac{n}{2}+\delta}(\sqrt{\lambda} r) r^{\frac{n}{2}-\delta-1} d r
$$

By the asymptotic formula (see [5; p.199])

$$
\begin{gathered}
J_{\nu}(s)=\left(\frac{2}{\pi s}\right)^{1 / 2} \cos \left[s-(2 \nu+1) \frac{\pi}{4}\right]+O\left(\frac{1}{s^{3 / 2}}\right), \\
v_{\lambda}^{\delta, R}(f, x)-f(x)=f(x) O\left(\sqrt{\lambda}^{\alpha-\delta-1}\right)
\end{gathered}
$$

as $\lambda \rightarrow \infty$. Therefore $\left\|s_{\lambda}^{\delta}(f)-f\right\|_{K}=o\left({\sqrt{\lambda^{\alpha-\delta}}}^{-}\right.$for $\delta>\alpha-1$.

Next we assume that $\left\|s_{\lambda}^{s}(f)-f\right\|_{K}=o(1 / \lambda)$ for a compact set $K$ in $D$. Let $\boldsymbol{\varphi}$ be an infinitely differentiable function whose support is contained in $K$. Then the integral

$$
\int_{\Omega} \lambda\left[s_{\lambda}^{\delta}(f, x)-f(x)\right] \varphi(x) d x
$$

tends to zero. But the last integral equals

$$
\sum_{k=1}^{\infty} \frac{\lambda}{\lambda_{k}}\left[\left(1-\frac{\lambda_{k}}{\lambda}\right)^{+\delta}-1\right] f_{k} \boldsymbol{\lambda}_{k} \boldsymbol{\varphi}_{k}
$$

where $(1-t)^{+}=\max (1-t, 0)$. Since $\Sigma \lambda_{k}\left|f_{k} \boldsymbol{\varphi}_{k}\right|<\infty$ and the function $\left[(1-t)^{+\delta}\right.$ $-1] / t_{\text {_ }}$ is bounded, the above sum tends to

$$
-\delta \sum_{k=1}^{\infty} f_{k} \lambda_{k} \boldsymbol{\varphi}_{k}=-\delta \int_{\Omega} f(x) \Delta \boldsymbol{\varphi}(x) d x=0
$$


By the arbitrariness of $\phi$, we conclude that $f$ is almost everywhere equal to a harmonic function in $K$. Thus $f$ is harmonic in $K$ or more strongly in $D$.

4. To treat (ii) we shall use the following lemma. We give a proof of it passing the Fourier transformation.

LEMMA 2. Let $f$ be a function in $L^{2}\left(\boldsymbol{R}^{n}\right)$ and $D$ be an open domain in $\boldsymbol{R}^{n}$. Suppose that $f$ is regulated in $D$ and that the Laplacian $\Delta f$ of $f$ in the sense of distribution is bounded in $D$. If the sphere of radius $r$ with the center at $x$ is contained in $D$, then we have

$$
\frac{1}{\omega_{n}} \int_{|\omega|=1} f(x-r \omega) d \omega-f(x)=2^{\frac{n-1}{2}} \Gamma\left(\frac{n}{2}\right) \int^{r} d s \int_{|y| \leq s} \Delta f(x-y) s^{-n+1} d y .
$$

ProOF. We may assume that $f$ is infinitely differentiable and rapidly decreasing approximating $f$ by such functions. Then the interchanges of integrations in the following calculations are legitimate.

Let $\hat{f}(\xi)$ be the Fourier transform of $f$, i. e.,

$$
\hat{f}(\xi)=\frac{1}{\sqrt{2 \pi^{n}}} \int_{R^{n}} f(x) e^{-i \xi x} d x
$$

By Fourier inversion formula

$$
f(x-r \omega)-f(x)=\frac{1}{\sqrt{2 \pi^{n}}} \int_{R^{n}} \hat{f}(\xi)\left[e^{-i r \omega \xi}-1\right] e^{i \xi x} d \xi
$$

Integrating on the unit sphere we get

$$
\begin{aligned}
\frac{1}{\omega_{n}} \int_{|\omega|=1} f(x-r \omega) d \omega & -f(x)=\frac{1}{\sqrt{2 \pi}} \int_{R^{n}} \hat{f}(\xi)\left[\frac{1}{\omega_{n}} \int_{|\omega|=1} e^{-i r \omega \xi} d \omega-1\right] e^{i \xi x} d \xi \\
= & \frac{1}{\sqrt{2 \pi^{n}}} \int_{R^{n}} \hat{f}(\xi)\left[2^{\frac{n-2}{2}} \Gamma\left(\frac{n}{2}\right) \frac{J^{\frac{n-2}{2}}(r|\xi|)}{(r|\xi|)^{\frac{n-2}{2}}}-1\right] e^{i \xi x} d \xi
\end{aligned}
$$

Now by the Lommel's formula $([5 ; \mathrm{p} .45])$ 


$$
\int \frac{J_{v+1}(\mu s)}{s^{v}} d s=\frac{-1}{\mu} \frac{J_{s}(\mu r)}{r^{v}}
$$

we have

$$
2^{\frac{n-2}{2}} \Gamma\left(\frac{n}{2}\right) \frac{1}{r^{2}} \int_{0}^{r} \frac{J \frac{n}{2}(s|\xi|)}{(s|\xi|)^{\frac{n}{2}}} s d s=-\frac{1}{(r|\xi|)^{2}}\left[2^{\frac{n-2}{2}} \Gamma\left(\frac{n}{2}\right) \frac{\frac{J-2}{2}(r|\xi|)}{(r|\xi|)^{\frac{n-8}{2}}}-1\right] .
$$

Its Fourier transform is, by Bochner's formula,

$$
\begin{aligned}
& 2^{\frac{n-2}{2}} \Gamma\left(\frac{n}{2}\right) \frac{1}{r^{2}} \int_{0}^{r} s d s \frac{1}{\sqrt{2 \pi^{n}}} \int_{R^{n}}^{J \frac{n}{2}(s|\xi| \xi \mid)^{\frac{n}{2}}} e^{i \xi x} d \xi \\
& =2^{\frac{n-2}{2}} \Gamma\left(\frac{n}{2}\right) \frac{1}{r^{2}} \int_{\theta}^{r} x_{s}(x) s^{-n+1} d s \text {, }
\end{aligned}
$$

where $\chi_{s}(x)$ is the characteristic function of the ball $\{x:|x| \leqq s\}$.

Since $\frac{1}{\sqrt{2 \pi}^{n}} \int_{R^{n}}|\xi|^{2} \hat{f}(\xi) e^{i \xi x} d \xi=-\Delta f(x)$, by the convolution relation

$$
\begin{aligned}
& \frac{1}{\omega_{n}} \int_{|\omega|=1} f(x-r \omega) d \omega-f(x) \\
= & 2^{n-2} \Gamma\left(\frac{n}{2}\right) \int_{0}^{r}\left(\int_{R^{n}} \Delta f(x-y) \chi_{s}(y) d y\right) s^{-n+1} d s .
\end{aligned}
$$

If the sphere of radius $r$ with the center at $x$ is contained in $D$, then the last term is dominated in absolute value by

$$
r^{2}\|\Delta f\|_{D} \frac{2^{\frac{n-2}{2}} \mathrm{\Gamma}\left(\frac{n}{2}\right)}{\sqrt{2 \pi}^{n}} \frac{1}{r^{2}} \int_{0}^{r}\left(\int_{R^{n}} \chi_{s}(y) d y\right) s^{-n+1} d s=\frac{1}{2 n} r^{2}\|\Delta f\|_{D} .
$$

Proof of (ii). Suppose $f \in L^{2}(\Omega)$ and $\Delta f$ in the sense of distribution is bounded in a compact set $K$ of $\Omega$. We prove that

$$
\left\|s_{\lambda}^{\tilde{z}}(f)-f\right\|_{K^{\prime}}=O(1 / \lambda)
$$

for a closed subset $K^{\prime}$ strictly contained in $K$.

By Lemma 1 it suffices to see that 


$$
\left\|v_{i}^{\delta, R}(f)-f\right\|_{K^{\prime}} \leqq A / \lambda
$$

for $\lambda>1$. To prove this inequality we chose $R$ so small that $0<R<\operatorname{dis}\left(K^{\prime \prime}, K^{c}\right)$. By the similar way to the case (i) we have

$$
\begin{aligned}
& v_{\lambda}^{\hat{o}, R}(f, x)-f(x)
\end{aligned}
$$

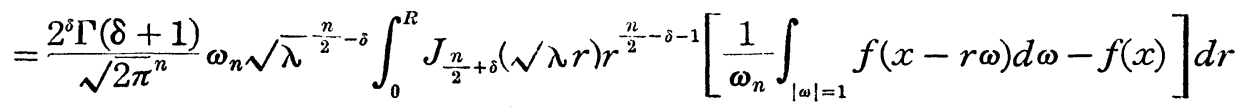

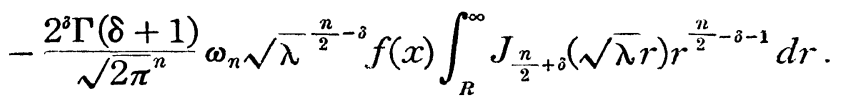

Since $\Delta f$ is bounded in $K$, so is $f$ in $K^{\prime}$. Thus the second term on the right hand side is $O\left(\sqrt{\lambda}^{\alpha-\delta-1}\right)$ by the same method as in (i).

The first term is, up to a constant multiple, equal to

$$
\begin{aligned}
& \sqrt{\lambda^{-\frac{n}{2}-\delta}} \int_{0}^{R} J_{\frac{n}{2}+\hat{\delta}}(\sqrt{\lambda} r) r^{\frac{n}{2}-\hat{o}-1} d r \int_{0}^{r} s^{-n+1} d s \int_{R^{n}} \Delta f(x-y) \chi_{s}(y) d y \\
& =\sqrt{\lambda^{-\frac{n}{2}-\delta}}\left(\int_{0}^{1 / \sqrt{\lambda}}+\int_{1 / \sqrt{\lambda}}^{R}\right) s^{-n+1} d s \int_{R^{n}} \Delta f(x-y) \chi_{s}(y) d y \int_{s}^{R} J_{\frac{n}{2}+\delta}(\sqrt{\lambda} r) r^{-\frac{n}{2}-\dot{\delta}-1} d r .(*)
\end{aligned}
$$

Changing a variable we get

$$
\int_{s}^{R} J_{\frac{n}{2}+\hat{\delta}}(\sqrt{\bar{\lambda}} r) r^{\frac{n}{2}-\delta-1} d r=\left(\frac{1}{\sqrt{\lambda}}\right)^{\frac{n}{2}-\delta} \int_{s \sqrt{\lambda}}^{R \sqrt{\lambda}} J_{\frac{n}{2}+\delta}(t) t^{\frac{n}{2}-\delta-1} d t .
$$

By the asymptotic formula before-mentioned the last term is $O\left(\sqrt{ } \lambda^{-\frac{3}{2}} s^{\alpha-\hat{\delta}-1}\right)$ if $\delta>\alpha-1$ and $s \sqrt{\lambda}>1$. Since $J_{\mu}(t)=O\left(t^{\mu}\right)$ as $t \rightarrow 0$, it is also $O\left(\sqrt{\lambda^{\delta-\frac{n}{2}}}\right)$ if $\delta>\alpha-1$ and $s \sqrt{ } \bar{\lambda} \leqq 1$. Thus (*) is dominated in $K^{\prime}$ by

$$
A \sqrt{\lambda^{-\frac{n}{2}-\hat{o}}}\|\Delta f\|_{K}\left(\int_{0}^{1 / \sqrt{\lambda}} s^{-n+1} s^{n} \sqrt{\lambda^{-\delta-\frac{n}{2}}} d s+\int_{1 / \sqrt{\lambda}}^{R} s^{-n+1} s^{n} s^{\alpha-\delta-1} \sqrt{ } \lambda^{-\frac{3}{2}} d s\right)
$$

which is not greater than $A\|\Delta f\|_{K} \sqrt{\bar{\lambda}^{\alpha-\delta-1}}$ if $\alpha+1>\delta>\alpha-1, \quad A\|\Delta f\|_{K} \lambda^{-1} \log \lambda$ if $\delta=\alpha+1$ and $A\|\Delta f\|_{K} \lambda^{-1}$ if $\delta>\alpha+1$ respectively.

Next we assume $\left\|s_{\lambda}^{i}(f)-f\right\|_{K}=O(1 / \lambda)$. For an infinitely differentiable function $\boldsymbol{\phi}$ whose support is contained in $K$, we have 


$$
-\delta \sum_{k=1}^{\infty} \lambda_{k} f_{k} \boldsymbol{\varphi}_{k} \leqq A\|\boldsymbol{\phi}\|_{L^{\prime}(K)}
$$

which is proved similarly to the case (i). Thus

$$
\mid\langle f, \Delta \varphi>|=|\langle\Delta f, \boldsymbol{\varphi}\rangle| \leqq A\|\boldsymbol{\varphi}\|_{L^{2}\left(K^{\prime}\right)} .
$$

Therefore $\Delta f$ is (essentially) bounded in $K$.

\section{REFERENCES}

[1] V. A. IL'IN, Problems in localization and convergence of Fourier series in fundamental systems of functions of the Laplace operator, Uspehi Mat. Nauk, 23(1968), 61-120; Russian Math. Surveys, 23(1968), 59-116.

[2] V. A. IL' IN, On the Riesz, Cesàro and Poisson-Abel summability of Fourier series in eigenfunctions, Differencial'nye Uravnenija, 2(1966), 816-827 (Russian).

[3] G. SuNOUCHI, Saturation in the local approximation, Tôhoku Math. J., 17(1965), 16-28.

[4] E. C. Titchmarsh, Eigenfunction Expansions Associated with Second-order Differential Equations, Oxford 1958.

[5] G. N. Watson, A Treatise on the Theory of Bessel Functions, Cambridge 1944.

MATHEMATICAI. INSTITUTE

TÔHOKU UNIVERSITY

SENDAI, JAPAN 